UM-P-95/113, RCHEP-95/25

\title{
POSSIBLE ELECTRIC CHARGE NONCONSERVATION AND DEQUANTIZATION IN $S U(2) \times U(1)$ MODELS WITH HARD SYMMETRY BREAKING
}

\author{
A.Yu.Ignatievt and G.C.Joshil \\ Research Centre for High Energy Physics, School of Physics, University of Melbourne, Parkville, \\ 3052, Victoria, Australia \\ (To appear in Physics Letters B)
}

\begin{abstract}
We study a novel type of extensions of the Standard Model which include a hard mass term for the U(1) gauge field and, optionally, the additional scalar multiplets spontaneously violating the electric charge conservation. Contrary to the case of abelian massive electrodynamics, in these theories the massiveness of photon necessarily implies non-conservation (and also dequantization) of the electric charge (even in the absence of spontaneous breakdown of the electromagnetic symmetry). On the other hand, unexpectedly, there exist models with charge non-conservation where it is possible to keep the photon mass zero (at least, at the tree level).
\end{abstract}

Typeset using REVTEX 
1. In the past, there have been many papers exploring the possibility that the photon may have non-zero mass [1]. At first, these works were made in the context of massive electrodynamics, which is an abelian $\mathrm{U}(1)$ theory with the added photon mass term $\frac{1}{2} m^{2} A_{\mu}^{2}$. The characteristic feature of such a theory is that the conservation of the electric charge is not violated by the presence of the photon mass term. The reason is that the photon mass term violates the local gauge invariance but not the global one. Thus, massive electrodynamics suggests that it it is possible to have a massive photon along with the exact conservation of the electric charge.

Later, there emerged another class of theories with massive photon: non-abelian gauge theories with electric charge non-conservation [2 9]. The primary emphasis of these works was not on the massiveness of photon, but on the study of the possible electric charge nonconservation in gauge theories. Yet the massiveness of photon appeared to be an automatic consequence of the violation of the electric charge conservation. One of the important discoveries made in those works was the close relation between the two ideas: electric charge (non)conservation and electric charge (de)quantization [2, 4, 10].

Given all previous works, one important question still remains unexplored: is it possible to have massive photon and exact electric charge conservation in realistic theories? Of course, it is possible within $\mathrm{U}(1)$ massive electrodynamics, but it is not a realistic theory. What we are interested in is this: can the Standard $S U(2) \times U(1)$ model be extended or modified in such a way as to have both the massive photon and the exact electric charge conservation simultaneously.

At first sight all we have to do is to give a hard mass to the $\mathrm{U}(1)$ gauge boson $B_{\mu}$. That would presumably make photon massive without spoiling the electric charge conservation. However, we will show that it turns out not to be the case.

A related but different question that we are going to consider is this: can we have $a$ massless photon in a theory with electric charge non-conservation? One reason to ask this question is the very stringent experimental bound on the photon mass: $m_{\gamma}<10^{-24} \mathrm{GeV}$ or even $10^{-36} \mathrm{GeV}$ [1]. This bound places a very tight constraints on any theory with electric 
charge non-conservation and one is naturally curious how to evade it.

Naively, one might think that the answer to the above question is negative. Yet in this work we will construct examples of realistic $S U(2) \times U(1)$ models in which the photon mass is zero at the tree level but the electric charge is not conserved ${ }^{1}$.

2.Let us first consider a model which differs from the Standard Model only in one point: its lagrangian contains a mass term for the $U(1)$ gauge field $B$ (before spontaneous symmetry breaking):

$$
\mathcal{L}^{\prime}=\mathcal{L}_{0}+\frac{1}{2} m^{2} B_{\mu}^{2}
$$

where $\mathcal{L}_{0}$ is the Standard Model lagrangian.

After spontaneous symmetry breaking, we diagonalize the gauge boson mass matrix and obtain the physical fields $\mathrm{A}$ and $\mathrm{Z}$ which we identify with the photon and Z-boson:

$$
\begin{aligned}
& A_{\mu}^{3}=Z_{\mu} \cos \theta^{\prime}+A_{\mu} \sin \theta^{\prime} \\
& B_{\mu}=A_{\mu} \cos \theta^{\prime}-Z_{\mu} \sin \theta^{\prime} .
\end{aligned}
$$

where the mixing angle $\sin ^{2} \theta^{\prime}$ is different from the Weinberg angle of the Standard Model $\left(\sin ^{2} \theta\right):$

$$
\sin ^{2} \theta^{\prime}=\sin ^{2} \theta+\frac{m^{2}}{M_{Z}^{2}}\left(1-\frac{e^{2}}{\sin ^{2} \theta}+e^{2}-\sin ^{4} \theta\right) \approx \sin ^{2} \theta+0.64 \frac{m^{2}}{M_{Z}^{2}}
$$

Note that the photon acquires a non-zero mass: $M_{\gamma}=g m$. Having obtained the mixing angle we can now write down the electromagnetic part of the lagrangian $\mathcal{L}^{\prime}$ :

$$
\begin{aligned}
\mathcal{L}^{\prime e m} & =\mathcal{L}_{l}^{\prime e m}+\mathcal{L}_{q}^{\prime e m} \\
\mathcal{L}_{l}^{\prime e m} & =A_{\mu}\left[\frac{1}{2}\left(g \sin \theta^{\prime}-g^{\prime} \cos \theta^{\prime}\right) \bar{\nu}_{L} \gamma^{\mu} \nu_{L}-\frac{1}{2}\left(g \sin \theta^{\prime}+g^{\prime} \cos \theta^{\prime}\right) \bar{e}_{L} \gamma^{\mu} e_{L}\right.
\end{aligned}
$$

\footnotetext{
${ }^{1}$ Note that the elaboration of these models beyond the tree level lies outside the scope of this paper. Thus at this stage we cannot rule out the possibility that the ultimate answer to the above question should be negative
} 


$$
\begin{aligned}
& \left.-g^{\prime} \cos \theta^{\prime} \bar{e}_{R} \gamma^{\mu} e_{R}\right] \\
\mathcal{L}_{q}^{\prime} e m= & A_{\mu}\left[\frac{1}{2} g \sin \theta^{\prime}\left(\bar{u}_{L} \gamma^{\mu} u_{L}-\bar{d}_{L} \gamma^{\mu} d_{L}\right)+g^{\prime} \cos \theta^{\prime}\left(\frac{1}{6} \bar{u}_{L} \gamma^{\mu} u_{L}+\frac{1}{6} \bar{d}_{L} \gamma^{\mu} d_{L}+\right.\right. \\
& \left.\left.\frac{2}{3} \bar{u}_{R} \gamma^{\mu} u_{R}-\frac{1}{3} \bar{d}_{R} \gamma^{\mu} d_{R}\right)\right],
\end{aligned}
$$

where $g$ and $g^{\prime}$ are $\mathrm{SU}(2)$ and $\mathrm{U}(1)$ coupling constants (the rest of the notation being selfexplanatory). Based on this formula, we can arrive at an important conclusion: as soon as the equality $g \sin \theta=g^{\prime} \cos \theta$ is broken, the electromagnetic current conservation is violated immediately. To avoid confusion, one essential point needs to be emphasized here. We have defined the electromagnetic current (and thereby the electric charge) as the current interacting with (i.e. standing in front of) the electromagnetic field $A_{\mu}$. Naturally, one can ask about the standard fermion electromagnetic current of the form

$$
j_{\mu}=e\left(-\bar{e} \gamma_{\mu} e+\frac{2}{3} \bar{u} \gamma_{\mu} u-\frac{1}{3} \bar{d} \gamma_{\mu} d\right)
$$

Although this current is still conserved in the present model, it unfortunately becomes devoid of physical meaning, because all physical processes and experiments are based on the interaction between the charges and electromagnetic fields; therefore in the framework of the present model we have to attach physical meaning and reserve the name "electromagnetic current" for the current of Eq.(6) and (7), rather than that of Eq. (8)

To summarise, this theory features three fundamental deviations from the Standard Model: massiveness of photon, the electric charge dequantization, and the electric charge non-conservation.

Considering the experimental limits on the parameter $m$ we note that the experimental upper bound on the photon mass gives, by far, the strongest constraint on the value of $m$. It has been established that the photon mass should be less than $10^{-24} \mathrm{GeV}$ or even $10^{-36}$ [11. Therefore, we find that the parameter $m$ cannot exceed $2 \times 10^{-24} \mathrm{GeV}$ or $2 \times 10^{-36}$ GeV. With such small values of the parameter $m$, the charge dequantization and charge nonconservation effects are expected to be too small to be observed. (for a detailed discussion of experimental constraints on models with electric charge dequantization, see [16]). 
Note that this model (with no fermions) was first suggested in Ref. [17] under the name of "hybrid model". The authors of Ref. [17] were motivated by the systematic search for renormalizable gauge models beyond the standard $S U(2) \times U(1)$ model. As concerns the renormalizability of the model which is certainly a very important issue, it has been proved in Ref. [17] that the theory posesses the property called tree unitarity which is a weaker property than renormalizability. We are not aware of any work which would further address the problem of renormalizability of this type of models (cf. Ref. [18]). Although it may appear to be of academical rather than phenomenological character, this work would certainly be very desirable because it would include or exclude a whole new class of gauge models from the set of renormaliz able gauge theories. (Note that we do not share the belief that nonrenormalizability of a theory automatically makes it physically uninteresting.)

3.Let us now add to the previous Lagrangian a piece containing the scalar singlet field $\phi_{1}$ with the electric charge $\epsilon$ (which coincides with the hypercharge in this case):

$$
\left.\mathcal{L}_{1}=\mathcal{L}_{0}+\frac{1}{2} m^{2} B^{2}+\mid\left(\partial_{\mu}-i \frac{g^{\prime}}{2} B_{\mu}\right) \epsilon_{1}\right)\left.\phi_{1}\right|^{2}+P\left(\phi_{1}, \phi\right)
$$

Now, assume that the field $\phi_{1}$ has non-zero vacuum expectation value $v_{1}:\left\langle\phi_{1}\right\rangle=v_{1}$. Then, after spontaneous symmetry breaking and performing the diagonalization as before, we obtain the mass of the physical photon to be: $M_{\gamma}^{2}=g^{2}\left(m^{2}+\frac{1}{2} g^{2} v_{1}^{2} \epsilon_{1}^{2}\right)$. Thus, the formula for the photon mass (squared) consists of two contributions: the first is proportional to $\mathrm{m}^{2}$ ("hard mass") and the second is proportional to $v_{1}^{2}$ ("soft mass"). Nothing seems to prevent us from considering negative value for $\mathrm{m}^{2}$ (without loss of generality, $v_{1}^{2}$ can be always made positive by an appropriate gauge transformation). Thus we are led to a very interesting possibility: to choose these two parameters in such a way that they exactly cancel each other so that the photon remains masslessit (at least, at the tree level):

\footnotetext{
${ }^{2}$ Here, we disregard a possible appearence of a Nambu-Goldstone boson. One may expect that its manifestations would be sufficiently suppressed, but even if they were not, the model could be modified in analogy with Ref. [8].
} 


$$
m^{2}+\frac{1}{2} g^{\prime 2} v_{1}^{2} \epsilon_{1}^{2}=0
$$

It can be shown that if the condition (10) is satisfied, the Z-boson mass is not shifted at all. Now, do we obtain the electric charge non-conservation or dequantization in the fermion sector, in analogy with the result of Section 2? Unfortunately, the answer is: no. The reason is this: the calculation of the Weinberg angle in this model (denoted by $\theta_{1}$ ) shows that this angle is exactly equal to the Weinberg angle of the Standard Model: $\sin ^{2} \theta_{1}=\sin ^{2} \theta$. Note that this exact equality has been obtained without assuming $m^{2}$ or $v_{1}^{2}$ to be small (but, of course, assuming that the condition of photon masslessness, Eq. (10) holds.) From this equality it follows that the fermion electromagnetic current in this model remains exactly the same as in the Standard Model: $j_{\mu}=e\left(-\bar{e} \gamma_{\mu} e+\frac{2}{3} \bar{u} \gamma_{\mu} u-\frac{1}{3} \bar{d} \gamma_{\mu} d\right)$. In other words any effects of the electric charge non-conservation or dequantization are absent in the fermion sector. Here we would like to stress an essential point: the absence of these effects in the fermion sector does not mean that they are absent altogether. One should not forget that giving the vacuum expectation to the charged scalar field $\phi_{1}$ leads to the electric charge non- conservation in the scalar sector. However, from the phenomenological point of view, these effects are much harder to observe. Such effects would be similar to those arising in a model with charged scalar field but without the $m^{2}$ term. Models of such type have been considered in the literature before and we do not intend to go into details here.

Thus we see that in the context of the model with the lagrangian (9), vanishing of the photon mass leads to vanishing effects of charge non-conservation and charge dequantization in the fermion sector (but not in the scalar sector ).

4.Let us now change the singlet into the scalar doublet, again violating $\mathrm{U}(1)$ symmetry; the rest of the model will be the same. Thus, the lagrangian of our new model reads:

$$
\mathcal{L}_{2}=\mathcal{L}_{0}+\frac{1}{2} m^{2} B^{2}+\left|\left(\partial_{\mu}-i g \frac{\tau^{a}}{2} A_{\mu}^{a}-i \frac{g^{\prime}}{2}\left(1+\epsilon_{2}\right) B_{\mu}\right) \phi_{2}\right|^{2}+P\left(\phi_{2}, \phi\right) .
$$

where the electric charges of the scalar doublet are: 


$$
Q\left(\phi_{2}\right)=\left(\begin{array}{c}
1+\frac{\epsilon_{2}}{2} \\
\frac{\epsilon_{2}}{2}
\end{array}\right) .
$$

We break the electromagnetic symmetry by assuming

$$
\left\langle\phi_{2}\right\rangle=\frac{1}{\sqrt{2}}\left(\begin{array}{c}
0 \\
v_{2}
\end{array}\right) .
$$

After the spontaneous breakdown of symmetry we can find out that the condition for the photon to be massless is:

$$
m^{2}+\frac{1}{4} \epsilon^{2} g^{\prime 2} \frac{v^{2} v_{2}^{2}}{v^{2}+v_{2}^{2}}=0 .
$$

This condition can be satisfied by assigning negative value either to $m^{2}$ or to $v_{2}^{2}$.

Assuming for simplicity that the vacuum expectation of the second doublet is much smaller than that of the Higgs doublet, we can write down this expression for the mixing angle:

$$
\sin ^{2} \theta_{2}=\sin ^{2} \theta\left(1+2 \epsilon_{2} \cos ^{2} \theta \frac{v_{2}^{2}}{v^{2}}\right)
$$

where $\theta$ is the Weinberg angle of the Standard Model. The electromagnetic interaction is now given by:

$$
\begin{aligned}
\mathcal{L}_{2}^{e m}= & \mathcal{L}_{2 l}^{e m}+\mathcal{L}_{2 q}^{e m} \\
\mathcal{L}_{2 l}^{e m}= & A_{\mu}\left[\frac{1}{2}\left(g \sin \theta_{2}-g^{\prime} \cos \theta_{2}\right) \bar{\nu}_{L} \gamma^{\mu} \nu_{L}-\frac{1}{2}\left(g \sin \theta_{2}+g^{\prime} \cos \theta_{2}\right) \bar{e}_{L} \gamma^{\mu} e_{L}\right. \\
& \left.-g^{\prime} \cos \theta_{2} \bar{e}_{R} \gamma^{\mu} e_{R}\right] \\
\mathcal{L}_{2 q}^{e m}= & A_{\mu}\left[\frac{1}{2} g \sin \theta_{2}\left(\bar{u}_{L} \gamma^{\mu} u_{L}-\bar{d}_{L} \gamma^{\mu} d_{L}\right)+g^{\prime} \cos \theta_{2}\left(\frac{1}{6} \bar{u}_{L} \gamma^{\mu} u_{L}+\frac{1}{6} \bar{d}_{L} \gamma^{\mu} d_{L}+\right.\right. \\
& \left.\left.\frac{2}{3} \bar{u}_{R} \gamma^{\mu} u_{R}-\frac{1}{3} \bar{d}_{R} \gamma^{\mu} d_{R}\right)\right]
\end{aligned}
$$

We see that the charge dequantization and charge non-conservation effects are controlled by the parameter $\delta=g \sin \theta_{2}-g^{\prime} \cos \theta_{2}$. This parameter measures the deviation of our theory from the Standard Model (in the latter $g \sin \theta-g^{\prime} \cos \theta=0$ ). Up to the terms of the order of $\frac{v_{2}^{2}}{v^{2}}$ we have: $\delta=e \epsilon_{2} \frac{v_{2}^{2}}{v^{2}}$. In terms of $\delta$ we can conveniently express the dequantized lepton 
and quark charges. The neutrino charge is: $Q_{\nu}=\frac{1}{4} \delta$. The axial electron charge is equal to: $Q_{e}^{5}=-\frac{1}{4} \delta$. Our normalization is such that the vector electron charge should coincide exactly with $-e$, without any corrections: $Q_{e}=-e$. The vector $\left(Q_{u}\right)$ and the axial $\left(Q_{u}^{5}\right)$ charges of u-quark are given by: $Q_{u}=\frac{2}{3} e+\frac{1}{12} \delta, Q_{u}^{5}=\frac{1}{4} \delta$. The charges of d-quark are equal to: $Q_{d}=-\frac{1}{3} e-\frac{1}{6} \delta, Q_{d}^{5}=-\frac{1}{4} \delta$. Consequently, the vector charge of the neutron is: $Q_{n}=$ $Q_{u}+2 Q_{d}=-\frac{1}{4} \delta$. The vector charge of the proton equals $Q_{p}=2 Q_{u}+Q_{d}=e$. Therefore, although the electric charge is dequantized in this model, nevertheless the following relations between the fermion charges hold true: $Q_{n}+Q_{\nu}=0 ; \quad Q_{p}+Q_{e}=0$.

From various experiments testing the validity of electric charge quantization we can infer the following upper bounds on the parameter $\delta$. From the upper bound ( [14,15]) on the (electron) neutrino charge: $\delta<4 \times 10^{-13}$ or $4 \times 10^{-17}$. From the constraint ( [12]) on the neutron electric charge: $\delta<4 \times 10^{-21}$. From the tests ( [13]) of the neutrality of atoms: $\delta<4 \times 10^{-18}$.

5.To summarize, we have analyzed the issues of electric charge (non)conservation and the photon mass in the context of a new class of extended $S U(2) \times U(1)$ models (the characteristic feature of the class being the inclusion of a hard mass term for the $\mathrm{U}(1)$ gauge field). We have shown that the massiveness of photon necessarily implies non-conservation (and also dequantization) of the electric charge (even in the absence of spontaneous breakdown of the electromagnetic symmetry). This situation is in contrast with the case of the abelian U(1) massive electrodynamics. On the other hand, we have demonstrated that even in models with non-conservation of the electric charge it is possible to keep the photon mass zero (at least, at the tree level).

The authors are grateful to R.Foot and R.Volkas for stimulating discussions. This work was supported in part by the Australian Research Council. 


\section{REFERENCES}

* e-mail:sasha@tauon.ph.unimelb.edu.au

$\dagger \quad$ e- mail:joshi@bradman.ph.unimelb.edu.au

[1] For a review, see A.S.Goldhaber and M.M.Nieto, Rev. Mod. Phys. 43 (1971) 277.

[2] A.Yu.Ignatiev, V.A.Kuzmin and M.E.Shaposhnikov, Phys. Lett. B84 (1979) 315.

[3] L.B.Okun and Ya.B.Zeldovich, Phys.Lett. B78 (1978) 597; M.B.Voloshin and L.B.Okun, Pisma v ZhETF, 28 (1978) 156.

[4] A.Yu.Ignatiev, V.A.Kuzmin and M.E.Shaposhnikov, in Proc. Int.Conf. Neutrino-79 v.2, p.488.

[5] M.Suzuki, Phys. Rev. D38 (1988) 1544.

[6] M.M.Tsypin, Yad. Fiz. 50 (1989) 431.

[7] K.S.Babu and R.N.Mohapatra, Phys. Rev.D42, 3866 (1990).

[8] M.Maruno, E.Takasugi and M.Tanaka, Progr.Theor.Phys. 86, 907 (1991); E.Takasugi and M.Tanaka, Phys.Rev. D44, 3706 (1991).

[9] R.N.Mohapatra and S.Nussinov, Int. J. Mod. Phys. A7, 3817 (1992)

[10] R.R.Foot,G.C.Joshi, H.Lew and R.R.Volkas, Mod.Phys.Lett. A5, 95 (1990); ibid. A5, 2721 (1990); X.-G.He, G.C.Joshi, H.Lew and R.R.Volkas, Phys.Rev.D43, R22 (1991); ibid. D44, 2118 (1991); X.-G.He, G.C.Joshi and B.H.J.McKellar, Europhysics Lett. 10, 709 (1989); K.S.Babu and R.N.Mohapatra, Phys.Rev.Lett. 63, 938 (1989); Phys.Rev. D41, 271 (1990); R.R.Foot, Mod.Phys.Lett. A6, 527 (1991); N.G.Deshpande, Oregon Report OITS-107 (1979) (unpublished); a review: R.R.Foot, H.Lew and R.R.Volkas, J.Phys.G 19, 361 (1993); E.Takasugi and M.Tanaka, Progr.Theor.Phys. 87, 679 (1992);

[11] Particle Data Group, Review of Particle Properties, Phys. Rev. D50 (1994) Part 1. 
[12] J.Baumann, R.Gahler, J.Kalus, and W.Mampe, Phys. Rev. D37, 3107 (1988); see also R.Gahler, J.Kalus, and W.Mampe, Phys. Rev. D25, 2887 (1982)

[13] M.Marinelli and G.Morpurgo, Phys. Lett. 137B, 439 (1984); see also J.C.Zorn, G.E.Chamberlin, and V.W.Hughes, Phys. Rev. 129, 2566 (1963); H.F.Dylla and J.G.King, Phys. Rev. A7, 1224 (1973)

[14] J.Bernstein, M.Ruderman and G.Feinberg, Phys.Rev. 132, 1227 (1963)

[15] G.Barbiellini and G.Cocconi, Nature 329, 21 (1987)

[16] K.S.Babu and R.R.Volkas, Phys. Rev. D46, 2764 (1992)

[17] J.M.Cornwall, D.N.Levin and G.Tiktopoulos, Phys. Rev. Lett. 32 (1973) 498; Phys. Rev. D10 (1974) 1145.

[18] R.Delbourgo, S.Twisk and G.Thompson, Int.J. Mod.Phys.A3, 435 (1988). 